\title{
Sometimes Surgery is the Only Way to Make a Diagnosis and Treat the Patient: A Case of Congenital Partial Absence of Pericardium
}

\author{
Phivos C Symeonides \\ ${ }^{1}$ Department of Cardiology, Hippocrateon Hospital, Nicosia, Cyprus
}

Received: 06/02/2017

Accepted: 21/03/2017

Published: 05/04/2017

How to cite this article: Symeonides PC. Sometimes surgery is the only way to make a diagnosis and treat the patient: a case of congenital partial absence of pericardium. EJCRIM 2017;4: doi:10.12890/2017_000593

Conflicts of Interests: The Author declares that there are no competing interests.

Acknowledgements: I would like to express my sincere gratitude to Dr. Eleftheriou Eleftherios and Christou Christos, cardiologists; Mr. Neil Moat, consultant cardiac surgeon; and Dr. Michael Gatzoulis, Dr. Jonathan Clague and Dr. Vias Markides, consultant electrophysiologists.

This article is licensed under a Commons Attribution Non-Commercial 4.0 License

\section{ABSTRACT}

Introduction: A 47-year-old Caucasian woman with a past medical history of multiple ablative procedures for supraventricular arrhythmias and pacemaker implantation presented with increasing shortness of breath. The initial working diagnosis of the team treating her was ablation-induced pulmonary stenosis, especially after the recording of increased flow velocities through the right lower pulmonary vein. Case presentation: The patient was alert and oriented, but obviously dyspnoeic. The vital signs were normal. The physical examination revealed a soft cardiac systolic murmur and the lungs were clear on auscultation. The electrocardiogram showed a pacemaker rhythm. The echocardiogram showed borderline normal global systolic function of the left ventricle and severe mitral regurgitation. The transoesophageal echocardiogram confirmed the above findings and revealed increased velocities through the right lower pulmonary vein. The working diagnosis of ablation-induced pulmonary stenosis was reinforced by the cardiac CT angiography. The patient was subsequently referred for surgical intervention. The intra-operative findings were both unexpected and impressive: congenital partial absence of the pericardium was responsible for herniation of the right chambers into the pleural space. Mitral regurgitation was attributed to failure of coaptation due to the very short surface of the leaflets. Extensive external fibrosis around the pulmonary veins caused the pulmonary vein stenosis.

Conclusion: The final diagnosis of a partial pericardial defect causing torsion and distortion of the heart chambers was made only at surgery. The consistent finding of pulmonary vein stenosis in the non-invasive modalities and the past medical history of ablations initially misleadingly led us to the assumption that they were related.

\section{LEARNING POINTS}

- The initial working diagnosis of ablation-induced pulmonary vein stenosis based on the patient's shortness of breath, severe mitral regurgitation, right lower pulmonary vein stenosis and past medical history of multiple ablative procedures, was incorrect.

- The finding that the cardiac silhouette was borderline displaced to the right, the mildly hypoplastic right lung and the borderline impairment of the global systolic function of the left ventricle, especially after coronary artery disease was ruled out, should have been given greater consideration.

- Cardiac computer tomographic images taken 7 years ago were used to assess the severity of the right inferior pulmonary vein stenosis; new medical technologies will provide even better diagnostic techniques.

\section{KEYWORDS}

Pericardial defect; pulmonary vein stenosis 


\section{BACKGROUND}

A 47-year-old Caucasian woman presented to our hospital with increasing shortness of breath on exertion and easy fatigue. The patient had an extended past medical history of palpitations and syncopal episodes. She had been diagnosed with supraventricular arrhythmias, for which she had undergone multiple ablative procedures. An AAl pacemaker was implanted in 2002, which was upgraded a year later to DDD-R. During the previous 6 months, her symptoms had progressively deteriorated and the patient was admitted for further evaluation and treatment.

\section{CASE PRESENTATION}

On admission, the patient was oriented and alert. The physical examination was, apart from a soft cardiac systolic murmur, relatively unremarkable and the vital signs were normal.

We proceeded with laboratory tests in order to clarify the aetiology of the patient's symptoms. The blood analyses were normal. The electrocardiogram showed a normal paced rhythm. The chest $\mathrm{x}$-ray revealed a normal heart silhouette and aortic arch, borderline displaced to the right. The right lung was very mildly hypoplastic. A symptom-limited exercise treadmill test showed moderately impaired exercise capability. Interrogation of the pacemaker revealed that there was no underlying rhythm and no evidence of complex arrhythmias.

The echocardiogram showed a normal-sized left ventricle (LV) with preserved diastolic indices and borderline impaired systolic function (LVEF 48\%) due to mild global hypokinesia. There was severe mitral regurgitation (MR) as well as mild tricuspid insufficiency (TI). The right ventricular systolic pressure (RVSP) was borderline elevated ( $40 \mathrm{mmHg}$ ). The aortic root, left atrium and right chamber dimensions were within normal limits. A trivial pericardial effusion was noted. Further work-up with a transoesophageal echocardiogram (TOE) clearly confirmed significant MR due to failure of coaptation of the mitral leaflets. The TI was indeed mild and seemed to correlate with the presence of the pacemaker leads. Most importantly, increased velocities were recorded through the right lower pulmonary vein (Fig. 1).

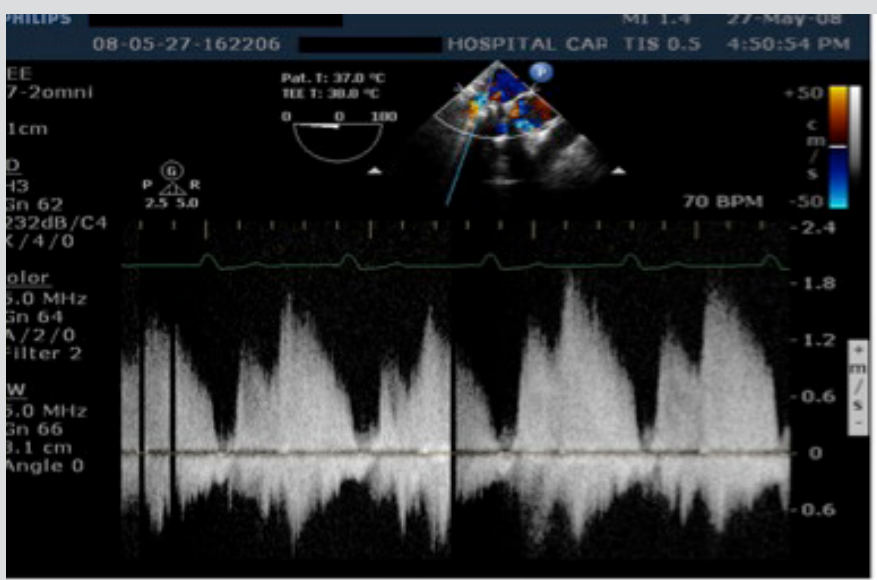

Figure 1. Transoesophageal echocardiogram (TOE): pulse wave Doppler recording of the flow at the entrance of the right inferior pulmonary vein. The normal flow pattern is two forward waves during systole and maximum velocities of $57 \pm 13 \mathrm{~cm} / \mathrm{sec}$ (mean $\pm S D$ ). However, in this case, increased velocities (up to $1.8 \mathrm{~m} / \mathrm{sec}$ ) indicative of significant intraluminar stenosis were seen

A presumptive working diagnosis of severe mitral insufficiency and ablation-induced stenosis of the right inferior pulmonary vein was made at that time. Further work-up with cardiac CT showed no evidence of coronary artery disease but clearly demonstrated total occlusion of the right inferior pulmonary vein (Figs. 2 and 3). In view of the above findings, the patient was referred to a specialized medical centre for mitral valve repair and reconstruction of the right inferior pulmonary vein.

Intra-operative TOE confirmed all the above findings. The operative findings, however, were impressive as well as unexpected. There was complete absence of the pericardium on the right side of the heart, with partial herniation of the right chambers in the pleural space. The pericardium was also absent on the left side of the heart. There was extensive external fibrosis around the pulmonary veins. The inferior pulmonary vein was a solid cord from about $1.5 \mathrm{~cm}$ away from the left atrium, and the rest of the pulmonary veins were also hypoplastic. This is markedly incompatible with post-ablation pulmonary stenosis. In the latter case, the pre-stenotic segment of the pulmonary vein is expected to be distended. Indeed, after the left atrium was opened, no evidence of scarring due to ablation was seen in that area. 

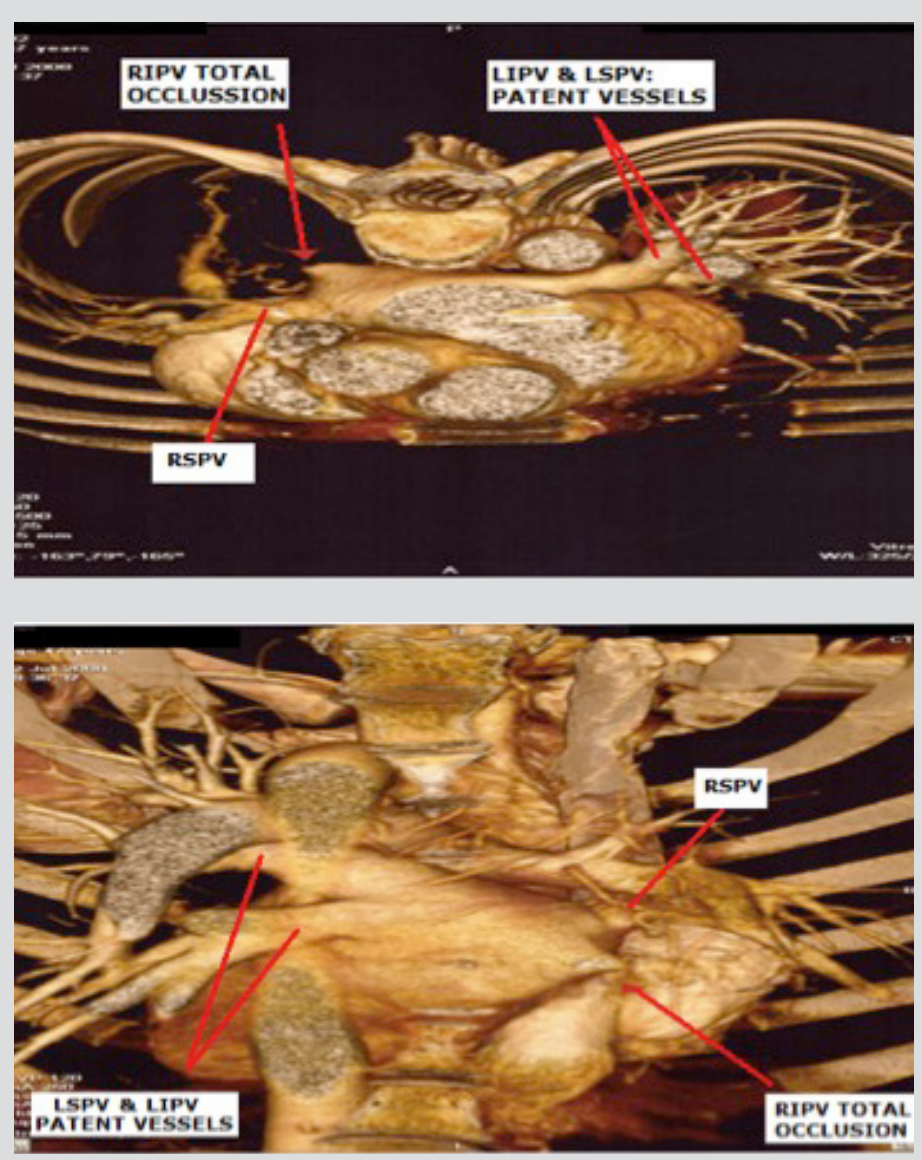

Figure 2. CT angiography: complete obstruction of the RIPV. The image shows the normal anatomy of the three pulmonary veins but complete obstruction of the RIPV

Figure 3. CT angiography: total occlusion of the RIPV. The image demonstrates the total occlusion of the RIPV. The other three pulmonary veins have a normal anatomy

LIPV, left inferior pulmonary vein; LSPV, left superior pulmonary vein; RIPV, right inferior pulmonary vein; RSPV, right superior pulmonary vein

\section{DISCUSSION}

This is a rare case of a patient who presented with shortness of breath and easy fatigue and diagnosed with pulmonary vein stenosis. It was at least plausible to initially connect the history of multiple ablations in the past with the pulmonary stenosis, especially after the findings of the TOE and the CT scan. However, it seems that despite ever more sophisticated imaging modalities, there are still cases where the final diagnosis is made in the operating theatre. It was a huge surprise when we realized that the pulmonary vein stenosis was due to extensive external fibrosis and hypoplasia. The chronic torsion of these vessels and the dislocation of the heart, as a result of the partially absent pericardium, were responsible for the hypoplastic changes. Congenital absence of the pericardium is an extremely uncommon finding, with a reported incidence of $0.01-0.02 \%$ in anatomopathological series ${ }^{[1]}$. Complete absence of the pericardium can allow the heart to shift within the thorax and can actually be asymptomatic, although exceedingly rare ${ }^{[2]}$. These patients are usually discovered incidentally during cardiac surgery for an unrelated condition or during a post-mortem investigation. Partial defects are usually left-sided. They can cause severe complications due to strangulation of the coronary arteries, valvular insufficiencies or traumatic injuries of the myocardium ${ }^{[3]}$. Partial pericardial defects may sometimes require surgical intervention to relieve the symptoms and/or complications. The most frequent complaints are non-exertional stabbing thoracic pain or discomfort that can sometimes be position-dependent and mimic coronary artery disease pain. Other symptoms include shortness of breath, palpitations and near-syncope ${ }^{[4]}$. The symptoms can sometimes be quite vague, although debilitating, and require a high index of suspicion for the diagnosis to be made. Heart MRI can provide excellent delineation of the pericardial anatomy and allow a thorough search of the entire chest for associated anomalies of the lungs and mediastinum. A registry from two hospitals describing congenital absence of the pericardium revealed that the most consistent diagnostic feature in MRI was a 'tongue' of lung tissue interposed between the main pulmonary artery and the aorta. In rare cases, thoracoscopic examination is needed to detect the malformation and estimate the risk of complications. 


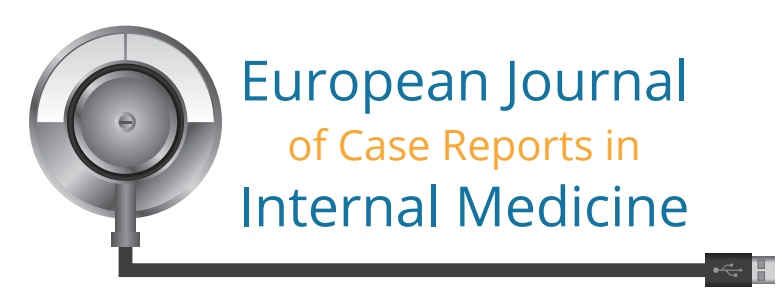

This particular patient underwent successful mitral valve repair. Seven years after the surgical procedure the patient was oligosymptomatic and experiencing a good quality of life.

\section{CONCLUSION}

This is a rare case of a middle-aged female who presented with increasing shortness of breath and easy fatigue. The initial working diagnosis of ablation-induced pulmonary vein stenosis was reinforced by the non-invasive findings of the TOE and the cardiac CT. Even the intra-operative TOE could not accurately predict the final diagnosis. The surgical procedure-and only that-uncovered impressive and unexpected findings and revealed the final diagnosis: partial absence of the pericardium. The subsequent torsion and distortion of the heart were responsible for the patient's symptoms. The surgical repair of the mitral valve relieved her symptoms and improved her quality of life.

\section{REFERENCES}

1. Wallace HW, Shen D, Baum S, et al. Angina pectoris associated with a pericardial defect. J Thorac Cardiovasc Surg 1971;61:461-465.

2. Abbas AE, Appleton CP, Liu PT, et al. Congenital absence of the pericardium: case presentation and review of literature. Int J Cardiol 2005;98:21-25.

3. Van Son JA, Danielson GK, Schaff HV, et al. Congenital partial and complete absence of the pericardium. Mayo Clin Proc 1993;68:743-747.

4. Gatzoulis MA, Munk MD, Merchant N, et al. Isolated congenital absence of the pericardium: clinical presentation, diagnosis, and management. Ann Thorac Surg 2000;69:12091215. 